\title{
Estado del conocimiento y prioridades de investigación sobre las familias Canidae, Mephitidae y Procyonidae (Mammalia: Carnivora) en Colombia
}

\author{
Gabriel Patricio Andrade-Ponce, ${ }^{1, *}$, Sandra Milena Montaño-Salazar², Luis Miguel Riveros-Loaiza', \\ Héctor E. Ramírez-Cháves ${ }^{3}$, Andrés Felipe Suárez-Castro ${ }^{4}$ \\ ${ }^{1}$ Grupo de mastozoología, Facultad de Ciencias, Universidad Nacional de Colombia, Bogotá, Colombia \\ 2 Facultad de Ciencias, Universidad Nacional de Colombia, Bogotá, Colombia \\ ${ }^{3}$ School of Biological Sciences, The University of Queensland, Brisbane, Queensland, Australia \\ ${ }^{4}$ School of Geography, Planning and Environmental Management, The University of Queensland, Brisbane, Queensland, Australia
}

\begin{abstract}
Resumen
A pesar de la gran diversidad de ecosistemas que habitan y de su importancia económica, ecológica y cultural, la información sobre las especies del orden Carnivora es escasa en Colombia. Con el fin de ejemplificar los sesgos y los avances en el conocimiento que se tiene sobre tres de las familias de Carnivora (Canidae, Mephitidae y Procyonidae) en el país, se evaluó el estado de la investigación sobre ellas con base en el número de publicaciones dedicadas a cada especie. Además, se estableció una escala de prioridades según el estado de amenaza y el esfuerzo de investigación de cada familia. Se encontraron 78 estudios sobre las tres familias y se constató que existen grandes vacíos relacionados con la ecología y la historia natural de la mayoría de las especies evaluadas, aun cuando el 80 \% de ellas se registraron por primera vez a nivel nacional hace más de 60 años. La mayoría de los estudios relacionados con la distribución y los registros correspondió a la familia Procyonidae, en tanto que la mayor parte de los estudios ecológicos se registró en torno a la familia Canidae, específicamente sobre la especie Cerdocyon thous. Aunque la prioridad más alta correspondió a Bassaricyon neblina, la mayoría de los estudios sobre las demás especies correspondió a evaluaciones locales en escalas temporales y espaciales pequeñas. El presente estudio muestra que la asignación de algunas especies a las diferentes categorías de amenaza se ha basado en supuestos erróneos que no han sido verificados en campo. Además, la presunta ausencia de ciertas especies en algunas provincias biogeográficas es señal de la existencia de grandes vacíos de información. Esta revisión sirve como marco de referencia para priorizar las investigaciones sobre los patrones de distribución y el estado de amenaza de las especies de estas familias en el país. (C) 2016. Acad. Colomb. Cienc. Ex. Fis. Nat.
\end{abstract}

Palabras clave: Carnívoros; Conservación; Prioridades de investigación.

State of knowledge and research priorities for Canidae, Mephitidae and Procyonidae (Mammalia: Carnivora) families in Colombia

\begin{abstract}
Despite the wide diversity of ecosystems inhabited by species of the order Carnivora, and their ecological, economic and cultural relevance, the information on the distribution, ecology, and taxonomy of these species in Colombia is limited. In order to show biases and advances on the state of knowledge of three families of Carnivora (Canidae, Mephitidae y Procyonidae) in the country, we evaluated research efforts based on the number of publications available for each species. In addition, we established a research priority scale based on how threatened they are and on the research effort for each species. We found a total of 78 studies in the country for the three families. There are research gaps related with ecology and natural history for most of the species, even though $80 \%$ of them were registered for the first time in Colombia more than 60 years ago. Most studies on distribution correspond to Procyonidae, whilst Canidae showed the largest number of ecological studies. However, these are mainly related with the crab-eating fox (Cerdocyon thous). Although Bassaricyon neblina had the highest research priority score, most of the studies for all the species are local assessments with small temporal and spatial scales. Our study shows that the assignment of the category of threatened to some species has been based on erroneous assumptions not verified in the field. In addition, the absence of some species in different biogeographic provinces is an indication of knowledge gaps. This review serves as a framework for prioritizing research associated with patterns of distribution and threatened status of the species of these families in the country. (c) 2016. Acad. Colomb. Cienc. Ex. Fis. Nat.
\end{abstract}

Key words: Carnivores; Conservation, Research priorities.

\footnotetext{
*Correspondencia:

Gabriel Patricio Andrade-Ponce, gpandradep@unal.edu.co; Recibido: 28 de febrero de 2016; Aceptado: 02 de agosto de 2016
} 


\section{Introducción}

Hasta el momento, en Colombia se han registrado siete familias (Canidae, Felidae, Mephitidae, Mustelidae, Otariidae, Procyonidae y Ursidae) y 33 especies del orden Carnivora (Suárez-Castro \& Ramírez-Cháves, 2015). Estas especies se distribuyen a lo largo de todas las regiones biogeográficas del país, desde el nivel del mar hasta los 4.100 m (Solari, et al., 2013). En los últimos años ha habido avances en cuanto al estado del conocimiento sobre este grupo de fauna en Colombia (Suárez-Castro \& Ramírez-Cháves, 2015). El establecimiento de prioridades de investigación (González-Maya, et al., 2011a), así como la recolección de datos en trabajos de campo (Delgado \& Zurc, 2007; Sánchez-Lalinde \& Pérez-Torres, 2008) y las colecciones biológicas (Marín, et al., 2012; Helgen, et al., 2013), han permitido entender la taxonomía, la ecología y los patrones de distribución de varias especies.

El desarrollo de dichos estudios ha permitido el registro del 89 \% (8 de 9) de las especies sudamericanas de la familia Procyonidae, el 45 \% (5 de 11) de la familia Canidae y el 33 \% (1 de 3) de la familia Mephitidae en el país (Hunter, 2011). Entre estas especies, grupos como el de los mapaches (Procyon spp.) y el de los olingos (Bassaricyon spp.) cuentan con revisiones taxonómicas recientes, en las cuales se destaca la descripción de una nueva especie con presencia en Colombia (Helgen, et al., 2013). Además, se han compilado nuevos registros de distribución para especies de coatíes (González-Maya, et al., 2011b) y cánidos (Ramírez-Cháves, et al., 2013).

A pesar de estos avances, no existe un análisis cuantitativo que permita determinar a ciencia cierta el estado del conocimiento sobre la ecología, la taxonomía o el estado de conservación de varias especies, aun cuando algunas de ellas son comunes y de amplia distribución (Suárez-Castro \& Ramírez-Cháves, 2015). Dado que el análisis sobre el estado del conocimiento en torno a un grupo es el primer paso para plantear investigaciones, así como planes de manejo y conservación acordes con las necesidades locales y regionales (Rodríguez-Mahecha, et al., 2006), en el presente trabajo se evaluó el esfuerzo de investigación con base en los diferentes temas tratados en las publicaciones dedicadas a cada especie nativa de las familias Canidae, Mephitidae y Procyonidae en Colombia. Además, se señalaron los vacíos de información y los avances de la investigación en los últimos años de acuerdo con las prioridades de investigación propuestas por otros autores (González-Maya, et al., 2011a). Se espera que esta información facilite la priorización de los proyectos de investigación teniendo en cuenta las limitaciones de tiempo y de recursos financieros y humanos.

\section{Materiales y métodos}

Se hizo una búsqueda de literatura (artículos científicos y libros) en torno a cada una de las especies de las familias Canidae, Mephitidae y Procyonidae presentes en
Colombia en el motor de búsqueda Web of Science (http:// thomsonreuters.com/), así como en trabajos de grado, tesis e informes técnicos. Para ello se emplearon los nombres de las familias, los géneros y Colombia como palabras clave en la búsqueda.

En cada fuente bibliográfica encontrada se determinó la especie o grupo de especies objetivo, el tema tratado y la provincia biogeográfica continental en la cual se realizó el estudio (sensu Morrone 2014: Chocó-Darién, Guajira, Magdalena, Sabana, Cauca, Ecuador occidental, Napo, Imerí y Páramo). Los temas estudiados se dividieron en siete categorías: 1) amenazas y relación con el ser humano; 2) anatomía y morfología; 3) distribución y registros; 4) ecología; 5) genética; 6) generalidades, y 7) taxonomía y sistemática (Figura 1).

Para establecer la escala de prioridad de la investigación se siguió la metodología de Cossios, et al. (2012) con algunas modificaciones. Cada especie recibió un puntaje comprendido entre cero y uno en cada una de las siguientes categorías: estado de amenaza y esfuerzo de investigación. La manera de asignar los valores a cada categoría fue la siguiente.

a) Estado de amenaza: con base en las categorías de la Unión Internacional para la Conservación de la Naturaleza - IUCN (2014), la Resolución 192 del 10 de febrero de 2014 del Ministerio de Ambiente y Desarrollo Sostenible - MADS, (2014), y los Apéndices de la Convención sobre el Comercio Internacional de Especies Amenazadas de Fauna y Flora Silvestres - CITES (2014), se asignó un valor al estado de amenaza. El valor máximo de esta categoría fue de uno, por lo cual cada subcategoría representaba una fracción del total. La lista roja de la UICN y las categorías de la Resolución 0192 del 2014 representaron 2/5 del total del puntaje de cada una, mientras que los apéndices del CITES representaron 1/5; esto con el fin de evitar la sobrevaloración de los puntajes de amenaza de especies en los apéndices I y II. El puntaje mínimo de cada subcategoría se estableció dividiendo el número de categorías de conservación (siete, incluida la categoría de 'no evaluada') sobre la fracción correspondiente, por lo que especies no evaluadas (NE) en la lista de la UICN y en la Resolución 192 recibieron un puntaje de 2/35. Conforme aumentaba la categoría de riesgo se sumaba el valor mínimo al valor de la categoría anterior. En el caso de los apéndices del CITES, el valor mínimo se obtuvo de la división de 1/5 en cuatro categorías, por lo cual una especie no listada en los apéndices recibía un puntaje de 1/20 (Tabla 1). Los tres puntajes obtenidos por cada especie en cada lista se sumaron para obtener el puntaje final. Así, una especie clasificada como "en peligro crítico (CR)” tanto por la IUCN (2014) como por la Resolución 192, así como en el Apéndice I del CITES, obtenía un puntaje final de uno, mientras que una especie no incluida en alguna de las categorías de conservación y en los apéndices del CITES obtenía un puntaje de 0,16 . 
b) Esfuerzo de investigación: el puntaje de esta categoría se asignó con base en la cantidad de temas estudiados para cada especie. En este apartado solo se tomaron en cuenta las listas de especies si presentaban datos no disponibles previamente en otras publicaciones. El máximo puntaje de esta categoría fue de uno y cada tema de la bibliografía obtuvo un valor de $1 / 7$, de modo que por cada tema en el cual una especie presentara al menos un estudio, se restaba 1/7 del valor máximo de esa categoría. Así, las especies sin información publicada en algún tema recibieron un puntaje de uno, mientras que las especies para las cuales se registró información en los siete temas obtuvieron un puntaje de cero (Tabla 2).

\section{Distribución y esfuerzo de investigación por provincia biogeográfica}

Con el fin de establecer el esfuerzo de investigación en diferentes regiones del país, se contó el número de estudios en cada provincia biogeográfica según el método de Morrone
(2014). Además, se determinó la presencia de cada especie en cada provincia a partir de la revisión de las siguientes bases de datos: Global Biodiversity Information Facility (GBIF, www.gbif.org/), VertNet (www.vertnet.org) y el Sistema de Información sobre Biodiversidad de Colombia (SiB, http://data.sibcolombia.net), en las cuales se obtuvo información sobre ejemplares procedentes de diez museos extranjeros (Anexo 1, http://www.raccefyn.co/index.php/ raccefyn/article/downloadSuppFile/327/1189). Asimismo, se obtuvo información de las especies depositadas en la colección de mamíferos del Instituto de Investigaciones Biológicas “Alexander von Humboldt” (IAvH), la Colección “Alberto Cadena García” del Instituto de Ciencias Naturales de la Universidad Nacional de Colombia (ICN), el Museo de Historia Natural de la Universidad de Nariño (PSO-CZ) y el Museo de Historia Natural de la Universidad del Cauca (MHNUC). Con el fin de complementar las localidades en provincias que no tenían registros conocidos con ejemplares

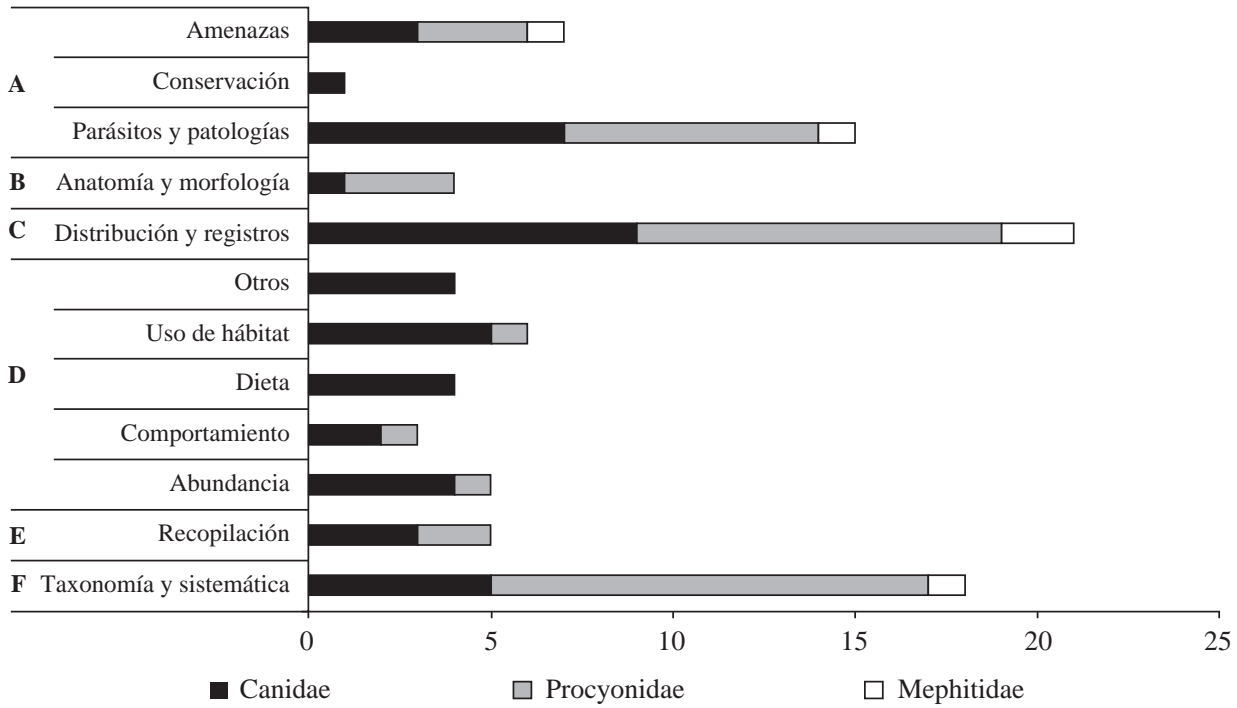

Figura 1. Número de estudios registrados sobre cada tema. Estudios registrados para cada categoría y subcategoría de temas con sus respectivas descripciones para las familias Canidae, Mephitidae y Procyonidae. A. Amenazas y relación con el ser humano. B. Anatomía y morfología. C. Distribución y registros. D. Ecología. E. Generalidades. F. Taxonomía y sistemática

Tabla 1. Puntaje por categoría de la variable de estado de amenaza según la Unión Internacional para la Conservación de la Naturaleza IUCN, la Resolución 192 del Ministerio de Ambiente, Vivienda y Desarrollo Territorial y la Convención sobre el Comercio Internacional de Especies Amenazadas de Fauna y Flora Silvestres - CITES. Su sumatoria da como resultado el puntaje final de la categoría de estado de amenaza para cada especie.

\begin{tabular}{lccccccc}
\hline Categorías según IUCN & CR & EN & VU & DD & NT & NC & No evaluado \\
\hline Puntaje & $2 / 5$ & $12 / 35$ & $2 / 7$ & $8 / 35$ & $6 / 35$ & $4 / 35$ & $2 / 35$ \\
Categorías según Resolución 192 & CR & EN & VU & DD & NT & NC & No evaluada \\
Puntaje & $2 / 5$ & $12 / 35$ & $2 / 7$ & $8 / 35$ & $6 / 35$ & $4 / 35$ & $2 / 35$ \\
Categorías de la CITES & \multicolumn{2}{c}{ Apéndice I } & \multicolumn{2}{c}{ Apéndice II } & & Apéndice III & No incluido \\
Puntaje & \multicolumn{2}{c}{$1 / 5$} & & $3 / 20$ & & $1 / 10$ & $1 / 20$ \\
\hline
\end{tabular}

Abreviaciones categoría de estado de amenaza: CR: En peligro crítico, EN: En peligro, VU: Vulnerable, DD: Datos deficientes, NT: Casi amenazada, NC: No considerada 
Tabla 2. Prioridades de investigación para las especies de las familias Canidae, Procyonidae y Mephitidae en Colombia. Escala de prioridades de investigación para cada especie y sus respectivos puntajes (P)

\begin{tabular}{|c|c|c|c|c|c|c|c|c|}
\hline \multirow[t]{2}{*}{ Familia } & \multirow[t]{2}{*}{ Especie } & \multicolumn{4}{|c|}{ Estado de amenaza } & \multicolumn{3}{|c|}{ Esfuerzo de investigación } \\
\hline & & Provincias habitadas & UICN & Res. 192 & CITES & $\mathbf{P}$ & Temas & $\mathbf{P}$ \\
\hline \multirow[t]{5}{*}{ Canidae } & Atelocynus microtis & Sabana, Imerí & $\mathrm{LC}$ & NE & II & 0,32 & $1,2,3,6$ & 0,43 \\
\hline & Cerdocyon thous & $\begin{array}{l}\text { Chocó-Darién, Guajira, Magdalena, } \\
\text { Sabana, Cauca, Imerí, Páramo }\end{array}$ & LC & NE & II & 0,32 & $1,2,3,4,6,7$ & 0,14 \\
\hline & Lycalopex culpaeus & Cauca & LC & VU & II & 0,55 & $1,2,3,6$ & 0,43 \\
\hline & Speothos venaticus & $\begin{array}{l}\text { Guajira, Magdalena, Sabana, } \\
\text { Cauca, Imerí, Napo }\end{array}$ & NT & NE & I & 0,43 & $2,3,4,6$ & 0,43 \\
\hline & $\begin{array}{l}\text { Urocyon } \\
\text { cinereoargenteus }\end{array}$ & $\begin{array}{l}\text { Guajira, Magdalena, Sabana, } \\
\text { Cauca, Páramo }\end{array}$ & $\mathrm{LC}$ & NE & NI & 0,22 & $1,2,4,6$ & 0,43 \\
\hline \multirow[t]{9}{*}{ Procyonidae } & Bassaricyon alleni & $\begin{array}{l}\text { Guajira, Magdalena, Sabana, } \\
\text { Imerí Páramo }\end{array}$ & $\mathrm{LC}$ & NE & NI & 0,22 & $2,6,7$ & 0,57 \\
\hline & Bassaricyon medius & Chocó- Darién, Magdalena, Cauca, & LC & $\mathrm{NE}$ & NI & 0,22 & $3,6,7$ & 0,57 \\
\hline & Bassaricyon neblina & Magdalena, Cauca, Páramo & $\mathrm{NT}^{*}$ & NE & NI & 0,27 & 6,7 & 0,71 \\
\hline & Nasua narica & Chocó-Darién, Cauca & $\mathrm{LC}$ & NE & NI & 0,22 & $3,6,7$ & 0,57 \\
\hline & Nasua паsua & $\begin{array}{l}\text { Magdalena, Sabana, Cauca, } \\
\text { Napo, Imerí, Páramo }\end{array}$ & LC & NE & II & 0,32 & $2,4,6,7$ & 0,43 \\
\hline & Nasuella olivacea & Magdalena, Cauca, Páramo & DD & NE & NI & 0,33 & $1,2,3,4,6,7$ & 0,14 \\
\hline & Potos flavus & $\begin{array}{l}\text { Chocó-Darién, Guajira, Magdalena, } \\
\text { Sabana, Cauca, Napo, Páramo }\end{array}$ & $\mathrm{LC}$ & NE & II & 0,32 & $2,4,5,6,7$ & 0,29 \\
\hline & $\begin{array}{l}\text { Procyon } \\
\text { cancrivorus }\end{array}$ & $\begin{array}{l}\text { Chocó-Darién, Guajira, Magdalena, } \\
\text { Sabana, Cauca, Ecuador occidental, Napo }\end{array}$ & LC & NE & NI & 0,22 & $2,3,6,7$ & 0,43 \\
\hline & Procyon lotor & Guajira & $\mathrm{LC}$ & $\mathrm{NE}$ & NI & 0,22 & $3,6,7$ & 0,57 \\
\hline Mephitidae & $\begin{array}{l}\text { Conepatus } \\
\text { semistriatus }\end{array}$ & Guajira, Magdalena, Sabana, Cauca, & $\mathrm{LC}$ & NE & NI & 0,22 & $1,6,7$ & 0,57 \\
\hline
\end{tabular}

- Categoría de amenaza UICN para B. neblina tomada de Helgen, et al. (2013).

de colección, se tuvieron en cuenta los registros novedosos publicados en revistas científicas y en inventarios que presentaban una localidad específica y evidencia sustentada en referencias (Anexo 1).

\section{Resultados}

\section{Estado del conocimiento}

Se encontraron 78 estudios sobre las tres familias en Colombia (Anexo 2, http://www.raccefyn.co/index.php/ raccefyn/article/downloadSuppFile/327/1189). De estos, el $74,3 \%$ correspondía a artículos científicos, $8,9 \%$ a libros y capítulos de libros, $6,4 \%$ a notas cortas, 5,1 \% a informes técnicos y 5,1\% a trabajos de grado y tesis. La familia con mayor número de estudios ( $\mathrm{n}=44$ ) fue Canidae (Figura 1), y la menos estudiada fue Mephitidae $(n=5)$. Entre los estudios encontrados para las tres familias, los temas más recurrentes se relacionaban con registros novedosos de distribución y ecología (Figura 1). Aunque la familia Procyonidae cuenta con más especies que la familia Canidae (nueve y cinco, respectivamente), solo tres de ellas (Nasuella olivacea, Nasua nasua, Potos flavus) aparecen mencionadas en más de cinco estudios (Figura 2). En cuanto a la abundancia de estudios por provincia (Anexo 2, Figura 3), Cauca fue la provincia con el mayor esfuerzo de investigación $(\mathrm{n}=25)$, seguida por Sabana $(n=16)$, Magdalena $(n=14)$, Guajira $(\mathrm{n}=10)$, Chocó-Darién y Páramo $(\mathrm{n}=6$ cada una), mientras que Imerí, Napo y Ecuador occidental fueron las menos estudiadas. A continuación se presentan breves sinopsis del estado del conocimiento y la distribución de cada una de las especies evaluadas.

\section{Familia Canidae}

Atelocynus microtis (Sclatter, 1882). Se encontraron registros provenientes de los departamentos de Amazonas, Caquetá, Guaviare y Meta, en las provincias de Sabana e Imerí (Figura 4). Se desconoce su presencia en otros departamentos de dichas provincias. A nivel nacional solo cuenta con estudios sobre su distribución (Hershkovitz, 1961; Defler \& Santacruz, 1994; Ayure \& González-Maya, 2014), abundancia relativa (Payán \& Escudero, 2016), horarios de actividad (Payán, 2009), endoparásitos (Thatcher, 1971), medidas craneales y externas (Andrade-Ponce, JiménezRamírez, Montaño-Salazar \& Riveros-Loaiza, 2015) y generalidades (Hershkovitz, 1957; Hershkovitz, 1961).

Cerdocyon thous (Linnaeus, 1766). Con base en los registros encontrados, la especie se distribuye en siete de nueve provincias consideradas para el país (Figura 4), y está 


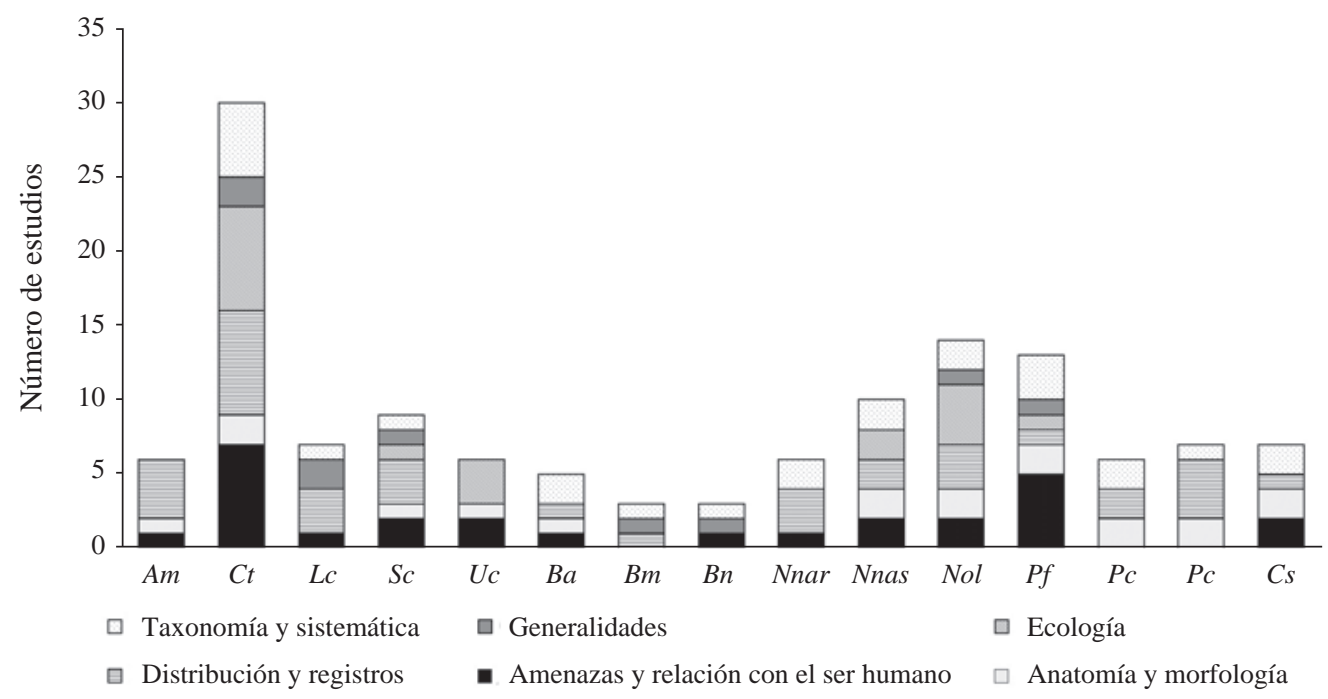

Figura 2. Publicaciones (artículos científicos, notas cortas, tesis de pregrado y capítulos de libros) encontradas sobre cada tema en las especies investigadas. Los totales no corresponden a la sumatoria de temas debido a que un estudio puede registrarse en diferentes categorías.

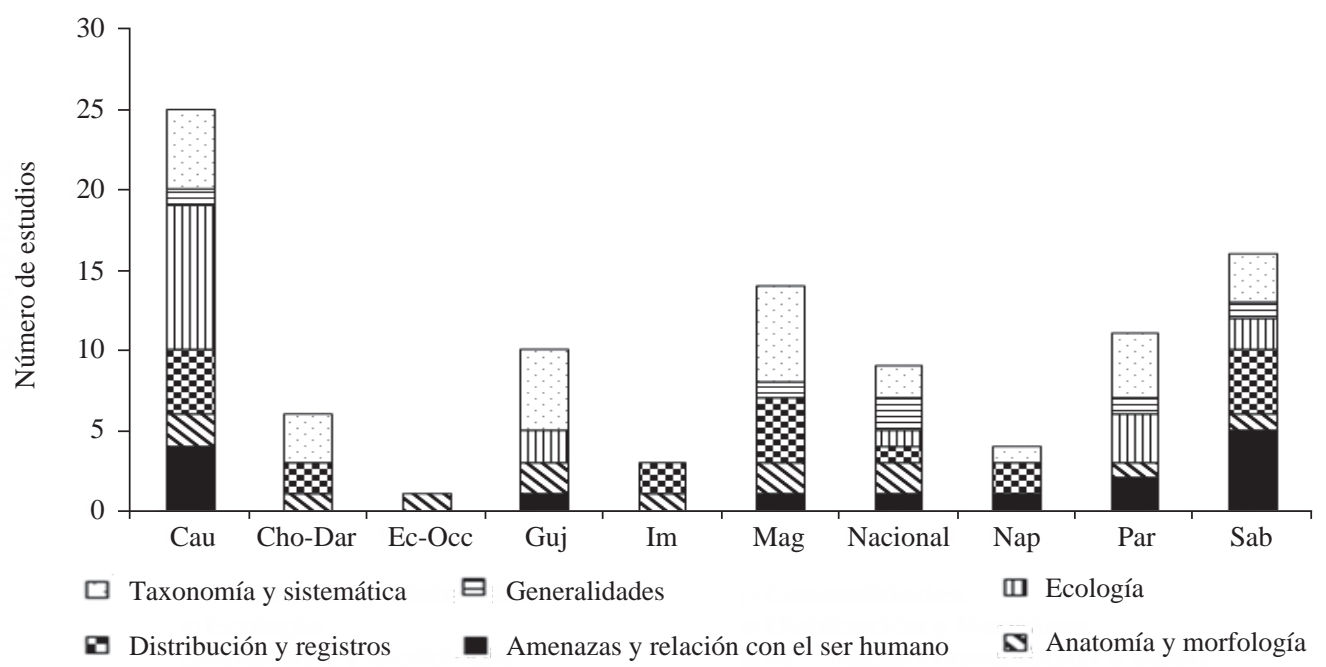

Figura 3. Número de estudios registrados para carnívoros de las familias Canidae, Mephitidae y Procyonidae en cada provincia biogeográfica de Colombia.

ausente en las provincias de Ecuador occidental y Napo, al suroccidente de Colombia. Los temas estudiados (Figura 2) incluyeron la caracterización de la dieta (Delgado-V, 2002; 2005; 2009; Delgado-V \& Zurc, 2007), las estimaciones de abundancia (Rodríguez-Rojas, 2005; Sánchez, et al., 2008; Boron \& Payán, 2013), el uso del hábitat (Martínez \& Cadena, 2000; Sánchez-Lalinde \& Pérez-Torres, 2008; Mejía-G. \& Londoño-S., 2012), el comportamiento (Sánchez-Londoño, 2014), los aspectos taxonómicos (Bangs,1898; Thomas, 1918; Allen, 1923), los ectoparásitos (Boshell \& Kerr, 1942; Wells, et al., 1981; Torres-Mejía \& De La Fuente, 2006), los endoparásitos y patógenos (Morales, et al., 1979; D’Alessandro, et al., 1981; 1984; Rojas-Arbeláez, et al., 2008), las medidas craneales y externas (Andrade-Ponce, et al., 2015), la distribución y las generalidades (Hershkovitz, 1957; Díaz-Pulido, et al., 2014, Pardo \& Payan, 2015; Ramírez-Cháves \& Pérez, 2015; Suárez-Castro, et al., 2015).

Lycalopex culpaeus (Molina, 1782). Esta especie presentó tres registros confirmados (Figura 4) que la restringen al departamento de Nariño y, por lo tanto, a la provincia de Cauca. Cuenta únicamente con estudios sobre su distribución (Hershkovitz, 1957, Ramírez-Chaves \& Noguera Urbano, 2011; Ramírez-Chaves, et al., 2013), su taxonomía (Hershkovitz, 1957), una revisión de la categoría de amenaza (Jorgenson, et al., 2006), las medidas craneales y externas (Andrade-Ponce, et al., 2015) y las generalidades (Hershkovitz, 1957; Jorgenson, et al., 2006) (Figura 2). 

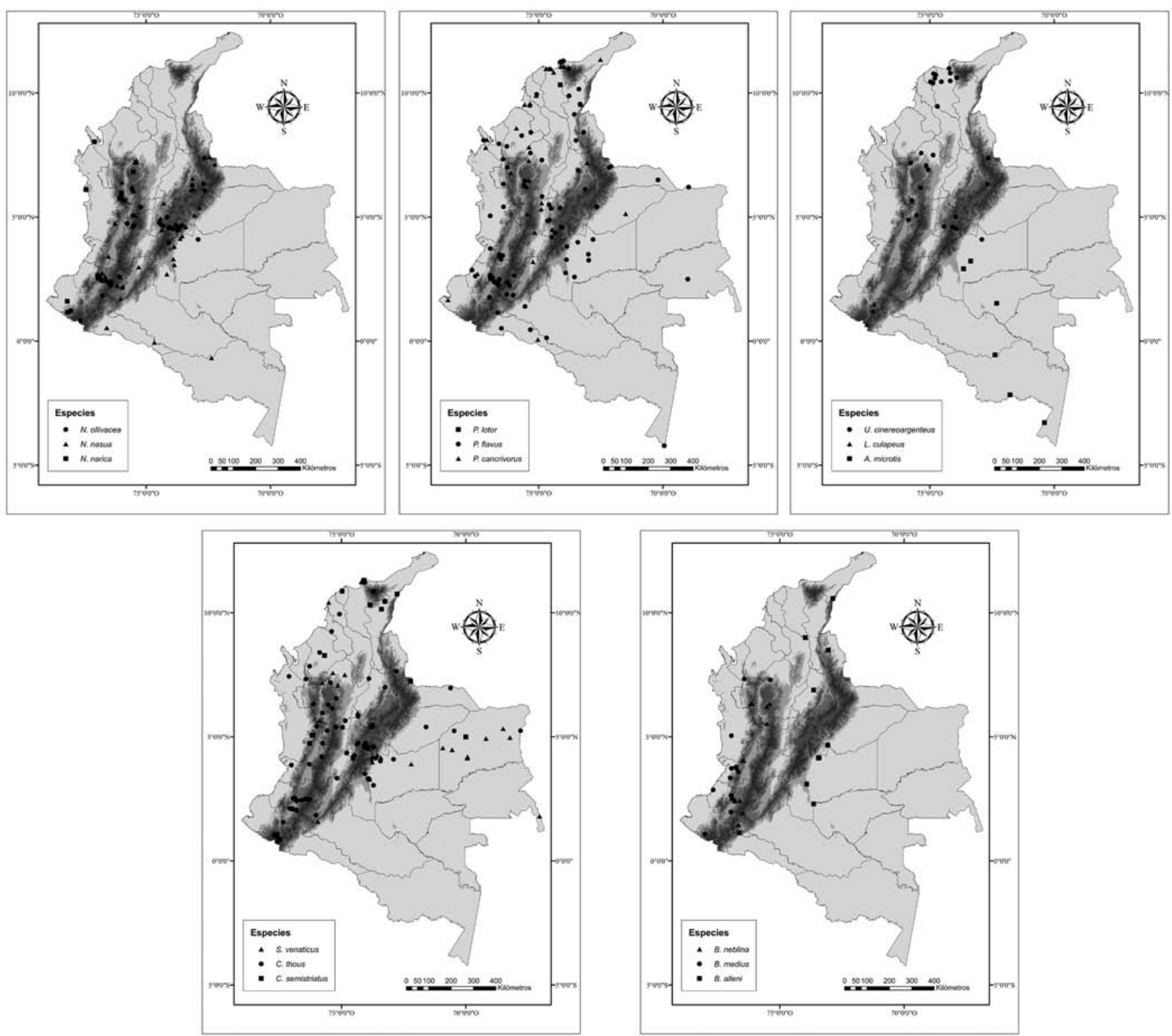

Figura 4. Mapas con localidades marginales para las especies de las familias Canidae, Mephitidae y Procyonidae en Colombia

Speothos venaticus (Lund, 1842). La especie cuenta con registros provenientes de las provincias de Guajira, Magdalena, Sabana, Amazonas, Cauca, Napo e Imerí (Figura 4). Los temas estudiados (Figura 2) incluyeron la distribución (Defler, 1986; De Armas-Mendoza, 2007; CarreteroPinzón, 2013; Negret, et al., 2015), su comportamiento en cautiverio (Bates, 1944), las medidas craneales (AndradePonce, et al., 2015), las amenazas por atropellamientos en las vías (De La Ossa-Nadjar \& De La Ossa, 2013), la taxonomía y las generalidades (Hershkovitz, 1957), así como un estudio sobre ectoparásitos (Boshell, et al., 1942).

Urocyon cinereoargenteus (Schreber, 1775). Se encontraron registros procedentes de las provincias de Guajira, Magdalena, Sabana, Cauca y Páramo (Figura 4). Los estudios abarcaron temas referentes a su abundancia y ecología
(Orjuela \& Jiménez, 2004; Baptiste, 2009; Mejía-G. \& Londoño-S., 2012), las medidas craneales y externas (Andrade-Ponce, et al., 2015), los patógenos (RojasArbeláez, et al., 2008), y la incidencia de la rabia (Páez, et al., 2005), aunque estos últimos registros pueden tratarse, en su mayoría, de casos en $C$. thous, ya que las fotos publicadas corresponden a esta especie (Suárez-Castro, et al., 2015).

\section{Familia Mephitidae}

Conepatus semistriatus (Boddaert, 1785). Se encontraron registros de esta especie distribuidos en las provincias de Guajira, Magdalena, Sabana y Cauca (Figura 4). La especie es mencionada en varias listas de mamíferos del país (Allen, 1916: como Conepatus mapurito; Moreno-Bejarano \& Álvarez-León, 2003; Solari, et al., 2013; Díaz-Pulido, et 
Tabla 3. Primeras menciones bibliográficas de las especies de las familias Canidae, Mephitidae y Procyonidae para Colombia

\begin{tabular}{|c|c|c|}
\hline Especie & Referencia & Comentarios \\
\hline A. microtis & Hershkovitz (1957) & \\
\hline C. thous & Bangs (1898) & $\begin{array}{l}\text { Bangs (1898) describió a Urocyon aquilus (= Cerdocyon thous aquilus), con } \\
\text { localidad tipo en Santa Marta }\end{array}$ \\
\hline L. culpaeus & Hershkovtiz (1957) & \\
\hline S. venaticus & Bates (1944) & \\
\hline U. cinereoargenteus & Hershkovitz (1957) & \\
\hline C. semistriatus & Howel (1906) & $\begin{array}{l}\text { Howel (1906), menciona que la especie fue descrita con base en observaciones } \\
\text { realizadas por Mutis. }\end{array}$ \\
\hline B. alleni & Thomas (1927) & $\begin{array}{l}\text { Thomas (1927) describió a Bassaricyon medius siccatus, que se considera como } \\
\text { sinónimo de B. alleni (Helgen, et al., 2013). }\end{array}$ \\
\hline B. medius & Thomas (1909) & $\begin{array}{l}\text { Thomas (1909) describió la especie basándose en un ejemplar procedente de } \\
\text { Colombia (BMNH 9.7.17.10, Jiménez, montañas de Chocó). }\end{array}$ \\
\hline B. neblina & Helgen, et al. (2013) & \\
\hline N. narica & Emmons \& Feer (1990) & \\
\hline N. nasua & Allen (1912), o Thomas (1912) & $\begin{array}{l}\text { Allen (1912) registró a Nasua quichua basado en un ejemplar proveniente de } \\
\text { Popayán. Thomas (1912) describió a Nasua candace, localidad en Medellín }\end{array}$ \\
\hline N. olivacea & Gray (1865) & Localidad tipo en los alrededores de Santa Fe de Bogotá \\
\hline P. flavus & Martin (1836), Allen (1904b) & $\begin{array}{l}\text { Martin (1836) describió a Cercoleptes megalotus, con localidad tipo desconocida. } \\
\text { Allen (1904b) consideró a C. megalotus como una subespecie de P. flavus, con } \\
\text { localidad tipo en Santa Marta, Colombia. }\end{array}$ \\
\hline P. cancrivorus & Allen (1904a) & $\begin{array}{l}\text { Descripción de Procyon proteus, localidad tipo en Bonda, Santa Marta, sinónimo de } \\
\text { P. cancrivorus. }\end{array}$ \\
\hline P. lotor & Cuervo-Díaz, et al. (1986). & \\
\hline
\end{tabular}

al., 2014), en un estudio sobre infecciones con Echinococcus (D’Alessandro, et al., 1981) y en uno sobre fauna víctima de atropellamientos (De la Ossa-Nadjar \& De la Ossa, 2013). Además, en la revisión taxonómica del género se mencionan algunas localidades en las que se han encontrado ejemplares colombianos (Dragoo, et al., 2003), y también se hallaron discusiones sobre otros aspectos taxonómicos (Howell, 1906; Ramírez-Cháves \& Noguera-Urbano, 2011).

\section{Familia Procyonidae}

Bassaricyon alleni (Thomas, 1880). Los registros encontrados proceden de las provincias de Guajira, Magdalena, Sabana, Napo, Imerí y Páramo (Figura 4). En la revisión de Helgen, et al. (2013), quien sinonimizó a B. siccatus (descrito a partir de un ejemplar colombiano por Thomas, 1927) con esta especie, se utilizaron ejemplares colombianos. Además, se han reportado medidas craneales y externas de ejemplares no incluidos en la revisión antes mencionada (Andrade-Ponce, et al., 2015) y registros de distribución en la Amazonia colombiana (Suárez-Castro \& RamírezCháves, 2015). La especie aparece mencionada en un estudio de parasitología (como Bassaricyon gabbi, Wells, et al., 1981).

Bassaricyon medius (Thomas, 1909). La especie cuenta con registros procedentes de las provincias de Chocó-Darién, Magdalena y Cauca (Anexo 1, Figura 4), y fue descrita a partir de ejemplares procedentes de Colombia
(Thomas, 1909; Tabla 3). Además, se la menciona en un estudio sobre la extensión de su distribución en el departamento del Valle del Cauca (registrada como $B$. gabbi; Saavedra-Rodríguez \& Velandia-Perilla, 2011). Asimismo, se han empleado ejemplares colombianos en colecciones extranjeras para la revisión del género (Helgen, et al., 2013).

Bassaricyon neblina (Helgen, et al., 2013). Se encontraron en total 20 registros (Anexo 1, Figura 4), de los cuales solo uno no se menciona en la descripción de la especie (Helgen, et al., 2013) y corresponde a un espécimen (ICN 3729) proveniente del departamento de Putumayo, lo que sitúa a la especie en las provincias de Magdalena, Cauca y Páramo (Figura 4). Solo existe un estudio que abarca temas relacionados con la taxonomía, la distribución y las amenazas (Helgen, et al., 2013).

Nasua narica (Linnaeus, 1766). Los registros encontrados provienen de los departamentos de Antioquia, Chocó y Nariño, en las provincias de Chocó-Darién y Cauca (Figura 4). Se encontraron tres estudios sobre su distribución y sus registros en el país (Emmons \& Feer, 1990; GonzálezMaya, et al., 2011b; Morales-Martínez, et al., 2015). Además, varios ejemplares colombianos se emplearon para la revisión taxonómica del género (Decker, 1991) y la revisión preliminar de la familia Procyonidae en el país (GuzmánLenis, 2004). 
Nasua nasua (Linnaeus, 1766). La especie se distribuye en las provincias de Magdalena, Sabana, Cauca, Napo, Imerí y Páramo (Figura 4). Los temas tratados (Figura 2) en los estudios encontrados incluían datos sobre su comportamiento (Castellanos, 1999), las estimaciones de la abundancia (Rodríguez-Rojas, 2005; Payán, 2009; Boron \& Payán, 2013; Pardo \& Payán, 2015), sobre la parasitología (D’Alessandro, et al., 1981; Torres-Mejía \& De La Fuente, 2006), los aspectos taxonómicos y sistemáticos (Allen, 1912; Thomas, 1912; Thomas, 1914, Decker, 1991; GuzmánLenis, 2004), la morfología (Decker, 1991; Andrade-Ponce, et al., 2015) y la distribución (Decker, 1991; MoralesMartínez, et al., 2015).

Nasuella olivacea (Gray, 1865). La distribución conocida para la especie abarca las provincias de Magdalena, Cauca y Páramo (Figura 4). Es la especie más estudiada de la familia Procyonidae (Figura 2). Los temas tratados en las publicaciones abordaban su ámbito de acción y hábitos alimenticios (Rodríguez, 1995), las relaciones tróficas (Rodríguez-Bolaños, et al., 2000), sus patrones de actividad y área de acción (Rodríguez-Bolaños, et al., 2003), su abundancia (Sánchez, et al., 2008), taxonomía y distribución (Gray, 1865; Allen, 1916; Guzmán-Lenis, 2004; Helgen, et al., 2009) y su morfología (Helgen, et al., 2009; AndradePonce, et al., 2015). Además, la especie se menciona en un estudio sobre atropellamiento de fauna (Delgado-V, 2007) y presas de pumas de páramo (Hernández-Guzmán, $\boldsymbol{e}$ al., 2011). El estado del conocimiento sobre la especie y su distribución en el país fueron evaluados recientemente por Balaguera-Reina, et al. (2009).

Potos flavus (Schreber, 1774). La especie presenta registros que proceden de todas las provincias biogeográficas de Colombia, con excepción de Ecuador occidental (Figura 4). Se encontraron 13 estudios realizados para el país (Figura 2) relacionados con la taxonomía, la distribución y la filogeografía (Martín, 1836; Guzmán-Lenis, 2004; Ruiz-García, et al., 2013), con su comportamiento en cautiverio (Morales \& Sánchez, 2000), la parasitología y los patógenos (Overstreet, 1970; Trapido \& Sanmartín, 1971; Morales, et al., 1979; D’Alessandro, et al., 1981; Wells, et al., 1981; D’Alessandro, et al., 1984, TorresMejía \& De La Fuente, 2006), y su morfología y anatomía (Hernández-Camacho, 1977; Andrade-Ponce, et al., 2015), así como una monografía sobre la biología general de la especie (Hernández-Camacho, 1977).

Procyon cancrivorus (G. Cuvier, 1798). Los registros encontrados para esta especie provienen de todas las provincias biogeográficas terrestres de Colombia, excepto Imerí y Páramo (Figura 4). Se encontraron estudios sobre su taxonomía (Allen, 1904a; Marín, et al., 2012), morfología y anatomía (Guzmán-Lenis, 2004; AndradePonce, et al., 2015), su distribución y registros (Marín, et al., 2012; Noguera-Urbano \& Ramírez-Cháves, 2015; Pardo \& Payán, 2015) y su abundancia relativa (Boron \& Payán, 2013).
Procyon lotor (Linneaus, 1758). La especie ha sido registrada en los departamentos de Bolívar, Magdalena y Sucre, todos dentro de la provincia de Guajira (Figura 4), y a pesar de aparecer mencionada en varias listas locales (Cuervo-Díaz, et al., 1986; Moreno-Bejarano \& ÁlvarezLeón, 2003), solo cuenta con datos cráneo-dentales y externos (Guzmán-Lenis, 2004; Marín, et al., 2012).

\section{Prioridades de la investigación}

En la familia Canidae, las especies con los valores más altos según las prioridades de la investigación dedicada al estado de amenaza (Tabla 2) fueron $L$. culpaeus $(0,55)$ y $S$. venaticus $(0,43)$, mientras que en términos de esfuerzo de investigación, $C$. thous ha sido la más ampliamente estudiada $(0,14)$. En la familia Procyonidae, $N$. olivacea $(0,33)$ y $N$. nasua $(0,32)$ presentaron los valores más altos en términos del estado de amenaza, mientras que $B$. neblina $(0,71)$ tuvo la mayor prioridad en términos de esfuerzo de investigación debido al escaso número de estudios encontrados (Tabla 2). En el caso de $C$. semistriatus, esta especie presenta valores intermedios en los dos aspectos evaluados en comparación con todas las especies de las familias Canidae y Procyonidae (Tabla 2).

\section{Discusión}

A pesar de los avances investigativos en torno a los grupos evaluados, los resultados de la revisión indican que aún existen vacíos importantes en el conocimiento de varias especies en Colombia. En lo relacionado con pequeños carnívoros de las familias Mephitidae y Procyonidae, se han definido varias prioridades de investigación asociadas a temas como los patrones de distribución, la ecología y las amenazas (González-Maya, et al., 2011), pero solo se ha llevado a cabo la verificación de especímenes y la confirmación de la presencia de especies de los géneros Procyon y Bassaricyon (Marín, et al., 2012; Helgen, et al., 2013).

Dado que las prioridades de investigación en términos del estado de amenaza y el esfuerzo de investigación representan diferentes tipos de objetivos, a continuación se presenta un análisis independiente de cada uno de estos aspectos.

\section{Esfuerzo de investigación}

El esfuerzo de investigación sobre las familias estudiadas ha sido heterogéneo. Los resultados indican que la mayoría de estudios sobre la familia Procyonidae se han relacionado con su distribución y registros, en tanto que la mayor parte de los estudios ecológicos ha correspondido a la familia Canidae (Figura 1). Sin embargo, existe un sesgo asociado a las investigaciones sobre $C$. thous, una especie común que puede habitar en los perímetros urbanos (Rodríguez, 2005; Sánchez, et al., 2008). Por el contrario, no se cuenta con información acerca de la ecología de especies como $L$. culpaeus y A. microtis en el país.

En la única valoración previa del estado del conocimiento en la cual se incluía el orden Carnivora en Colombia (Stevenson, et al., 2006), se señalaba que las 
familias Procyonidae y Felidae eran las más estudiadas a nivel nacional, seguidas por la familia Canidae, aunque, infortunadamente, estos autores no reportaron el número exacto de trabajos revisados. Sin embargo, desde la fecha de publicación de dicho estudio, el incremento en el número de estudios ha sido mayor para Canidae (23) que para la familia Procyonidae (15; Anexo 2). Aun cuando se ha encontrado que las especies de menor tamaño tienden a ser menos estudiadas (Oliveira, 2006), en este caso los factores que inciden en el estado del conocimiento sobre las familias evaluadas fueron heterogéneos. Aspectos como el acceso a regiones remotas y los cambios taxonómicos recientes han afectado la distribución del esfuerzo de investigación en estos grupos. Por ejemplo, hay pocos estudios sobre especies como $A$. microtis (Amazonia) y N. narica (Chocó biogeográfico), que habitan regiones de difícil acceso.

De forma similar, Bassaricyon spp. han sufrido cambios taxonómicos en los últimos años, por lo que son escasos los estudios sobre las especies de este género recientemente descritas. Sirva de ejemplo B. neblina, la cual obtuvo el mayor puntaje de prioridad en esta categoría debido a que la información sobre ella solo proviene del trabajo de Helgen, et al. (2013) (Tabla 2).

La mayoría de los estudios sobre las demás especies correspondió a evaluaciones puntuales con escalas temporales y espaciales pequeñas. En general, no se registraron estudios sobre el estado de sus poblaciones a nivel nacional, ni sobre las relaciones ecológicas en áreas de simpatría, especialmente de los géneros Nasuella, Nasua y Conepatus (González-Maya, et al., 2011a). En este sentido, es necesario adelantar actividades de monitorización a largo plazo que permitan determinar las variaciones temporales en los patrones de abundancia y distribución de todas las especies (Walker, et al., 2000).

Por otro lado, se requieren valoraciones morfológicas o genéticas para determinar los patrones de variación intraespecífica (por ejemplo en Bassaricyon spp.), o como en el caso del género Nasuella, que permitan determinar caracteres precisos para confirmar la posible presencia en el país de N. meridensis (González-Maya, et al., 2011). Además, es necesario hacer revisiones adicionales para géneros como Procyon y Nasua, con el fin de determinar patrones de variación externa que permitan discriminar entre especies del género, de manera que funcionen como herramienta para evaluar su distribución en el país (González-Maya, et al., 2011a).

\section{Estado de amenaza}

Aunque la opinión de los expertos es un componente esencial para determinar el estado de amenaza de las especies, en muchas ocasiones existen sesgos que limitan la precisión de dicha aproximación (Regan, et al., 2005). La carencia generalizada de estudios básicos sobre las familias Canidae, Mephitidae y Procyonidae influye en la capacidad de valorar el estado de amenaza de algunas de sus especies a nivel nacional. La escasa documentación sobre la distribución de las especies y su estado poblacional ha conducido a un gran desconocimiento del impacto de las áreas protegidas en el mantenimiento y la perpetuidad de estas especies.

A pesar de que la disminución del hábitat y la cacería indiscriminada representan las principales amenazas que se ciernen sobre las especies del orden Carnivora (RodríguezMahecha, et al., 2011a), no hay datos compilados en torno a estos aspectos que permitan precisar cuáles poblaciones son las más vulnerables. Por otra parte, los estudios sobre la relación entre las amenazas, la salud, y el ser humano, considerados de la máxima prioridad en la investigación sobre pequeños carnívoros (González-Maya, et al., 2011a), han sido los menos frecuentes (Figura 1). Sin estos estudios es difícil determinar los factores importantes para la mitigación de riesgos o presiones negativas sobre las especies (Rodríguez-Mahecha, et al., 2006; Cossios, et al., 2012), información necesaria para integrar de manera eficaz estas especies en planes de manejo y conservación (SuárezCastro \& Ramírez-Chaves, 2015).

Aunque ninguna de las especies de estas tres familias se encuentra en la categoría de amenaza según la IUCN (2014), algunas de ellas deben ser valoradas de manera urgente. B. neblina es una de ellas, pues es una especie descrita recientemente. Hasta el momento, se ha sugerido incluirla en la categoría de “casi amenazada (NT)” debido a la pérdida de hábitat (Helgen, et al., 2013). En cuanto a N. olivacea, una de las especies más estudiadas en Colombia si se la compara con las demás especies de las familias evaluadas, la prioridad de la investigación es alta en cuanto al estado de conservación, ya que se la ha incluido en la categoría de "datos insuficientes" (DD) a nivel global. A nivel nacional, se han abordado aproximaciones para la reevaluación de la categoría de esta especie, y se ha sugerido clasificarla como “casi amenazada (NT)” debido a la disminución en su área de ocupación producto de la pérdida de hábitat, así como a su cacería como alimento y para usar su piel, y a la persecución a que es sometida por considerarla en algunos sitios como peste (Balaguera-Reina, et al., 2009).

En la familia Canidae, el lobo colorado (L. culpaeus) es la única especie categorizada como "vulnerable (VU)" tanto en el libro rojo de las especies amenazadas de mamíferos de Colombia (Rodríguez-Mahecha, et al., 2006) como en la Resolución 192 del 10 de febrero de 2014 (MAVDT, 2014), debido a la cacería, el tráfico de pieles y la pérdida y transformación de su hábitat (Jorgenson, et al., 2006). Sin embargo, Ramírez-Cháves, et al., (2013) sugirieron una reevaluación de la categoría de amenaza, debido a que se desconoce la información sobre los criterios utilizados anteriormente en el país, y a los registros recientes de la especie en el departamento de Nariño. Este ejemplo en particular demuestra que en varias ocasiones la clasificación de estas especies en las diferentes categorías de amenaza se ha basado en supuestos que requieren una recopilación de información mucho más detallada en campo, así como el consenso de un mayor número de asesores (Regan, et al., 2005). 


\section{Distribución}

Las diferencias en el número de investigaciones por provincias biogeográficas se deben, en parte, al papel de algunos centros investigativos de dichas regiones (Stevenson, et al., 2006) en el fortalecimiento del conocimiento sobre la diversidad, en este caso, de las provincias de Cauca, Páramo y Sabana, las cuales presentaron el mayor número de estudios (Figura 3). A pesar de los avances recientes en el estudio de la distribución de algunas de las especies de las familias evaluadas en este trabajo (BalagueraReina, et al., 2009; Marín, et al., 2012; Helgen, et al., 2013; Ramírez-Cháves, et al., 2013; Ramírez-Cháves \& Pérez, 2015; Suárez-Castro \& Ramírez-Cháves, 2015; Noguera-Urbano \& Ramírez-Cháves, 2015), aún es necesario hacer nuevas evaluaciones en campo y revisar las colecciones regionales para clarificar la distribución nacional biogeográfica y política de varias especies.

En las provincias de Imerí y Ecuador occidental se requieren inventarios más exhaustivos, ya que la ausencia de registros de algunas especies en estas zonas se debe, principalmente, a la falta de muestreos. Este es el caso de $P$. flavus, que no ha sido registrada en la provincia de Ecuador occidental en Colombia, pero sí en esta provincia del Ecuador (Ford \& Hoffmann, 1988).

La presente revisión también permitió incluir algunos registros omitidos en listas recientes (Solari, et al., 2013). La distribución de $U$. cinereoargenteus se extiende hacia la región de la Orinoquia, a partir de ejemplares procedentes de Puerto López, Meta (ICN 3222) y los llanos orientales (ICN 342). Además, existe un registro procedente de Dabeiba, Antioquia, (ICN 816) cuya localidad se ubica a una altura de $450 \mathrm{~m}$, muy por debajo del intervalo altitudinal mencionado por otros autores (Solari, et al., 2013; Suárez-Castro \& Ramírez-Cháves, 2015). Algunos registros históricos (Allen, 1916) respaldan la inclusión de $N$. olivacea en departamentos como Quindío (American Museum of Natural History [AMNH 33045-33049]) y Huila (MHNUC-M00098). Asimismo, no ha sido posible confirmar algunos registros de otras especies mencionados en la literatura científica. Este es el caso de S. venaticus, cuya distribución se ha sugerido para la región Pacífica (Muñoz-Saba \& Alberico, 2004; Solari, et al., 2013), a pesar de que no existe evidencia que permita confirmar esta información.

\section{Conclusiones}

Existen grandes vacíos de información sobre la ecología y la historia natural de la mayoría de las especies evaluadas (Suárez-Castro \& Ramírez-Cháves, 2015), a pesar de que gran parte de ellas (80 \%) fueron registradas a nivel nacional por primera vez hace más de 60 años (Tabla 3). Incluso con respecto a las especies más estudiadas como C. thous y $N$. olivácea, la información sobre temas de importancia para la conservación como la ecología básica, la distribución y las amenazas es escasa (Suárez-Castro
\& Ramírez-Cháves, 2015). Es importante resaltar el papel que vienen cumpliendo los centros e instituciones de investigación en el avance del conocimiento, ya que la mayoría de los estudios se centran en ubicaciones de provincias como Cauca, Sabana y Magdalena donde se concentran las instituciones y centros de investigación más grandes del país. Por lo tanto, el fortalecimiento de las entidades regionales es fundamental para cubrir los vacíos de información reportados en este trabajo. Además, la información generada a partir del conocimiento tradicional puede ayudar a consolidar aquella relacionada con el comportamiento, la historia de vida o la ecología de las especies evaluadas (Ramírez-Cháves, et al., 2008, Saavedra-Rodríguez \& Velandia-Perilla, 2011). Por último, se espera que este trabajo sirva como marco de referencia para priorizar la elaboración de planes de manejo y monitorización, así como las investigaciones sobre los patrones de distribución y el estado de amenaza de estas familias en el país.

Debe aclararse, asimismo, que el enfoque utilizado para evaluar las prioridades de investigación tuvo un componente de subjetividad, dada la elección hecha y el peso de los valores escogidos. Sin embargo, dicha metodología ofrece una visión general del estado del conocimiento en torno a las especies estudiadas, lo cual permite utilizar los resultados obtenidos en la adopción de decisiones sobre las estrategias de conservación y en la definición del enfoque de futuras investigaciones.

\section{Información suplementaria}

Anexo 1. Lista de registros de las especies de las familias Canidae, Mephitidae y Procyonidae, con sus respectivas fuentes y localidades. Acrónimos de las instituciones con ejemplares registrados. Vea el anexo 1 en: http://www.raccefyn.co/index.php/ raccefyn/article/downloadSuppFile/327/1189

Anexo 2. Lista de estudios registrados para las especies de las familias Canidae, Mephitidae y Procyonidae en Colombia, divididos por categorías y provincias. Vea el anexo 2 en: http://www.raccefyn.co/index.php/raccefyn/article/download SuppFile/327/1189

\section{Agradecimientos}

A las personas e instituciones que nos permitieron el acceso a la información sobre las familias evaluadas en el presente trabajo, entre quienes se destacan Hugo López (ICN), Claudia Medina, Socorro Sierra (IAvH) y Pilar Rivas (MHNUC). Al grupo de mastozoología de la Universidad Nacional de Colombia. Héctor E. Ramírez-Cháves agradece las becas concedidas por los programas Centennial Scholarship y Graduate School International Travel Awards de la Universidad de Queensland, y por la Universidad 
Quetzalcóatl durante la elaboración de este documento. Andrés Felipe Suárez-Castro agradece a Colciencias y a su programa de becas para estudios de doctorado en el exterior.

\section{Conflicto de intereses}

Los autores declaran no tener conflictos de intereses.

\section{Referencias}

Alberico, M., Cadena, A., Hernández-Camacho, J., MuñozSaba, J. (2000). Mamíferos (Synapsida: Theria) de Colombia. Biota Colombiana. 1 (1): 43-75.

Allen, G.M. (1923). The pampa fox of the Bogota Savanna. Proceedings of the Biological Society of Washington. 36: 55-58.

Allen, J.A. (1904a). New mammals from Venezuela and Colombia. Bulletin of the American Museum of Natural History. 20: 327-335.

Allen, J. A. (1904b). Mammals from Southern México and Central and South America. Bulletin of the American Museum of Natural History. 20: 30-80.

Allen, J.A. (1912). Mammals from Western Colombia. Bulletin of the American Museum of Natural History. 31: 71-95.

Allen, J.A. (1916). List of mammals collected in Colombia by the American Museum of Natural History expeditions, 1910-1915. Bulletin of the American Museum of Natural History. 25: 191-238.

Andrade-Ponce, G.P., Jiménez-Ramírez, J.S., Montaño-Salazar, S.M., Riveros-Loaiza, L.M. (2015). Generalidades del orden Carnivora. En Suárez-Castro, A.F. \& RamírezChaves (editores). Los carnívoros terrestres y semiacuáticos continentales de Colombia. Guía de campo (p. 23-25). Bogotá: Editorial Universidad Nacional de Colombia.

Ayure, S. \& González-Maya, J.F. (2014). Registro notable del perro de orejas cortas Atelocynus microtis (Carnivora: Canidae) en el trapecio amazónico, Colombia. Mammalogy Notes. 1 (1): 6-7.

Balaguera-Reina, S., Cepeda, A., Zárrate-Charry, D., GonzálezMaya, J. (2009). The state of knowledge of Western Mountain Coati Nasuella olivacea in Colombia, and extent of occurrence in the Northern Andes. Small Carnivore Conservation. 41: 35-40.

Bangs, O. (1898). Description of a new fox from Santa Marta, Colombia. Proceedings of the Biological Society of Washington. 12: 93-94.

Baptiste, M.P. (2009). Abundancia y ecología básica del zorro plateado (Urocyon cinereoargenteus) en el área de la planta de cemento Holcim, Nobsa, Boyacá. IAVH - 01352. 40 p.

Boron, V. \& Payán, E. (2013). Abundancia de carnívoros en el agropaisaje de las plantaciones de palma de aceite del valle medio del río Magdalena, Colombia. En C. Castaño-Uribe, J. F. González-Maya, C. Ange, D. Zárrate-Charry \& M. Vela-Vargas (editores). Plan de Conservación de Felinos del Caribe Colombiano, 2007-2012: los felinos y su papel en la planificación regional integral basada en especies clave. p. 165-176). Santa Marta: Fundación Herencia Ambiental Caribe, ProCAT Colombia, The Sierra to Sea Institute.

Boshell, J. \& Kerr, J. A. (1942). Veinticinco especies nuevas de Trombidiideos en Colombia. Revista de la Academia Colombiana de Ciencias Exactas, Físicas y Naturales. 5 (17): 110-117.
Bates, M. (1944). Notes on captive Icticyon. Journal of Mammalogy. 25: 152-154.

Cáceres-Martínez, C.H., Acevedo-Rincón, A.A., SánchezMontaño, L.R. (2015). Registros de plásticos en la ingesta de Tremarctos ornatus (Carnívora: Ursidae) y de Nasuella olivacea (Carnívora: Procyonidae) en el Parque Nacional Natural Tamá, Colombia. Revista Mexicana de Biodiversidad. 86 (3): 839-842.

Carretero-Pinzón, X. (2013). Bush dog sighting in a large forest fragment in the Colombian Llanos. Canid Biology and Conservation. 15: 16-17.

Castellanos, O. (1999). Breves observaciones etológicas del Nasua nasua (Procyonidae) en la reserva forestal Rioblanco, Manizales, Caldas. Boletín Científico, Centro de Museos, Museo de Historia Natural, Universidad de Caldas. 3: 53-55.

Cossíos, E.D., Alcázar, P., Fajardo, U., Chávez, K., AlfaroShigueto, J., Cárdenas-Alayza, S., Valqui, J., Montero, F.G., Lescano, J., Quevedo, M., Vivar, E., Leite, R., Ledesma, K., Medina, C., Maffei, L., Amanzo, J., Chávez, C., Enciso, A. García, M.A., Mangel, J.C., Mendoza, J.A., Rojas, G., Silva, L., Villegas, L., Williams, R. S.R., Zúñiga, A., Cruz, A., IMAPRE, Ruiz, E., DGFFSS. (2012). El orden Carnivora (Mammalia) en el Perú: estado del conocimiento y prioridades de investigación para su conservación. Revista Peruana de Biología. 19 (1): 017-026.

Cuartas-Calle, C. A. \& Muñoz-Arango, J. (2003). Lista de mamíferos (Mammalia: Theria) del departamento de Antioquia, Colombia. Biota Colombiana. 4 (1): 65-78.

Cuervo-Díaz, A., Hernández-Camacho, J., Cadena, G. (1986). Lista actualizada de mamíferos de Colombia. Anotaciones sobre su distribución. Caldasia. 15 (71-75): 471-502.

D’Alessandro, A., Barreto, P., Saravia, N., Barreto, M. (1984). Epidemiology of Trypanosoma cruzi in the Oriental plains of Colombia. American Journal of Tropical Medicine and Hygiene. 33 (6): 1084-1095.

D’Alessandro, A., Rausch, R.L., Morales, G. A., Collet, S, Ángel, S. (1981). Echinococcus infections in Colombian animals. American Journal of Tropical Medicine and Hygiene. 30 (6): 1263-1276.

De Armas-Mendoza, E. (2007). Levantamiento preliminar de medianos y grandes mamíferos no voladores en dos localidades del municipio de Arauca (Colombia). Tesis de pregrado. Universidad Nacional de Colombia, Sede Orinoquia.

De la Ossa-Nadjar, O. \& De la Ossa V.J. (2013). Fauna silvestre atropellada en dos vías principales que rodean los Montes de María, Sucre, Colombia. Revista Colombiana de Ciencias Animal. 5 (1): 158-164.

Defler, T.R. \& Santacruz, A. (1994). A capture of and some notes on Atelocynus microtis (Sclater, 1883) (Carnivora: Canidae) in the Colombian Amazon. Trianea. 5: 417-419.

Defler, T.R. (1986). A bush dog (Speothos venaticus) pack in the eastern Llanos of Colombia. Journal of Mammalogy. 67: 421-422.

Decker D. M. (1991).Systematics of the Coatis, genus Nasua (Mammalia, Procyonidae). Proceedings of the Biological Society of Washington. 104: 370-386.

Delgado-V., C.A. (2002). Food habits and habitat of the crab-eating fox Cerdocyon thous in the highlands of eastern Antioquia, Cordillera Central, Colombia. Mammalia. 66: 599-602.

Delgado-V., C.A. (2005). New record of Olallamys albicauda (Rodentia: Ehimydae) in Antioquia, Colombia. Brenesia. 64: 131-132. 
Delgado-V., C.A. (2007). Muerte de mamíferos por vehículos en la vía del Escobero, Envigado. Actualidades Biológicas. 29: 235-239.

Delgado-V., C.A. (2009). Depredación de Neusticomys monticolus (Rodentia: Sigmodontinae) por Cerdocyon thous (Carnivora: Canidae). Brenesia, 71 (72): 77-78.

Delgado-V., C.A. \& Zurc, D. (2007). Diet of the Crab-eating fox Cerdocyon thous (Carnivora: Canidae) in the Páramo de Belmira, Antioquia, Colombia. Brenesia. 67: 73-74.

Díaz-Pulido, A., A. Benítez, D. Gómez-Ruiz, C. CalderónAcevedo, A. Link, A. Pardo, F. Forero, G. De Luna, E. Payán, Solari, S. (2014). Mamíferos del bosque seco: una mirada al caribe colombiano. En: Pizano, C y H. García (editores). El bosque seco tropical en Colombia. p. 128-165. Bogotá: Instituto de Investigación de Recursos Biológicos Alexander von Humboldt (IAvH).

Dragoo, J.W., Honeycutt, L.R., Schmidly, D.J. (2003). Taxonomic status of white-backed hog-nosed skunks, genus Conepatus (Carnivora: Mephitidae). Journal of Mammalogy. 84: 159-176.

Emmons, L. H. \& Feer, F. (1990). Neotropical rainforest mammals: A field guide. Chicago, USA: University of Chicago Press.

Ford, L.S., \& Hoffmann, R.S. (1988). Potos flavus. Mammalian Species. 1988 (321): 1-9.

González-Maya, J.F., Cepeda, A.A., Belant, J.L., ZárrateCharry, D.A., Balaguera-Reina, S.A., RodríguezBolaños, A. (2011a). Research priorities for the small carnivores of Colombia. Small Carnivore Conservation. 44: 7-13.

González-Maya, J.F., Rodríguez-Bolaños, A., Pinto, D., JiménezOrtega, A. (2011b). Recent confirmed records and distribution of the white-nosed coati Nasua narica in Colombia. Small Carnivore Conservation. 45: 26-30.

Gray, J.E. (1865). A revision of the genera and species of ursine animals (Ursidae) founded on the collection in the British Museum. Proceedings of the Zoological Society of London. 1864: 677-709.

Guzmán-Lenis A.R. (2004). Revisión preliminar de la familia Procyonidae en Colombia. Acta Biológica Colombiana. 9: 69-76.

Helgen, K.M., Kays, R., Helgen, L., Tsuchiya-Jerep, M.T.N., Pinto, M., Koepfli, K.P, Eizirik, E., Maldonado, J. (2009). Taxonomic boundaries and geographic distributions revealed by an integrative systematic overview of the mountain coatis, Nasuella (Carnivora: Procyonidae). Small Carnivore Conservation. 41: 65-74.

Helgen, K.M., Pinto, C.M, Kays, R., Helgen, L.E., TsuchiyaJerep, M.T.N., Quinn, A., Wilson, D.E., Maldonado, J.E. (2013).Taxonomic revision of the Olingos (Bassaricyon), with description of a new species, the Olinguito. ZooKeys. 324: 1-83.

Hernández-Camacho, J. (1977). Notas para una monografía de Potos flavus (Mammalia: Carnivora) en Colombia. Caldasia. 11: 147-181.

Hernández-Guzmán, A., Payán, E., Monroy-Vilchis, O. (2011). Hábitos alimentarios de Puma concolor (Carnivora: Felidae) en el Parque Nacional Natural Puracé, Colombia. International Journal of Tropical Biology. 59: 1285-1294.

Hershkovitz, P. (1957). A synopsis of the wild dogs of Colombia. Novedades Colombianas, Museo de Historia Natural, Universidad del Cauca. 3: 157-161
Hershkovitz, P. (1961). On the South American small-eared zorro Atelocynus microtis Sclater (Canidae). Fieldiana Zoology. 39: 505-523.

Howell, A. H. (1906). The proper name for the white-backed skunk of Colombia. Proceedings of the Biological Society of Washington. 19: 45.

Hunter L. (2011). Carnivores of the world. Princeton: Princeton University Press. 240 p.

Unión Internacional para la Conservación de la Naturaleza IUCN. 2014. The IUCN Red List of Threatened Species. Version 2014.2. Disponible en: http://www.iucnredlist.org. Fecha de consulta: 6 de septiembre, 2014.

Jorgenson, J.P., Rodríguez-Mahecha, J.V., Constantino, E., Barrera de Jorgenson, A. (2006). Lobo colorado Lycalopex culpaeus. En: Rodríguez-Mahecha, J. V., Alberico, M., Trujillo, F. \& Jorgenson, J. (editores). Libro rojo de los mamíferos de Colombia. p. 237-241. Bogotá: Conservación Internacional Colombia, Ministerio de Ambiente, Vivienda y Desarrollo Territorial.

Marín, D., Ramírez-Chaves, H.E., Suárez-Castro, A.F. (2012). Revisión cráneo-dentaria de Procyon (Carnivora: Procyonidae) en Colombia y Ecuador, con notas sobre su taxonomía y distribución. Mastozoología Neotropical. 19: 259-270.

Martin, W. (1836). Description of two new species of the genus Cercolepetes. Proceedings of the Zoological Society of London. 1836: 81-83.

Martínez, Y. \& Cadena, A. (2000). Caracterización, evaluación y uso de hábitats del zorro perruno (Cerdocyon thous) en los llanos orientales de Colombia. Revista de la Academia Colombiana de Ciencias Exactas, Físicas y Naturales. 24: 383-391.

Ministerio de Ambiente y Desarrollo Sostenible - MADS. (2014). Resolución No. 192, "Por la cual se declaran las especies silvestres que se encuentran amenazadas en el territorio nacional y se toman otras determinaciones”. 25 de septiembre de 2010. 29 p.

Mejía-G., A. \& Londoño-S., P. (2012). Uso del hábitat e importancia de los parches de bosque y corredores ribereños para carnívoros en un paisaje fragmentado en la Sierra Nevada de Santa Marta, Colombia. Mastozoología Neotropical. 19: 179-195.

Morales G.A, Guzmán, E.A., Wells, E.A., Ángel, D. (1979). Polycystic echinococcosis in Colombia: The larval cestodes in infected rodents. Journal of Wildlife Diseases. 15: 421-428.

Morales A. \& Sánchez F. (2000). Efecto del enriquecimiento ambiental sobre el comportamiento de los perros de monte (Potos flavus) en el centro de recepción de fauna silvestre del Dama - Engativá. Documento preliminar.

Moreno-Bejarano, L.M. \& Álvarez-León, R. (2003). Fauna asociada a los manglares y otros humedales en el delta estuarino del río Magdalena, Colombia. Revista de la Academia Colombiana de Ciencias Exactas, Físicas y Naturales. 27: 517-534.

Muñoz-Saba, Y. \& Alberico, M. (2004). Mamíferos en el Chocó biogeográfico. En: Rangel-Ch., O. (editores).Colombia. Diversidad Biótica IV. El Chocó Biogeográfico/Costa Pacífica. p. 559-597. Bogotá D.C: Universidad Nacional de Colombia Instituto de Ciencias Naturales. 
Morales-Martínez, D.M, Suárez-Castro, A.F., CárdenasGonzález, C., Fernández-Rodríguez, C. (2015). Familia Procyonidae. En Suárez-Castro, A.F. \& Ramírez-Chaves (editores). Los carnívoros terrestres y semiacuáticos continentales de Colombia. Guía de campo. p. 134-165. Bogotá: Editorial Universidad Nacional de Colombia.

Negret, P.J., Garzón, O., Stevenson, P.R. (2015) First preliminary inventory of non-flying mammals of the Alto Fragua IndiWasi National Park, Colombia. Mammalogy Notes. 2: 32-35.

Noguera-Urbano, E.A. \& Ramírez-Cháves, H.E. (2015). Confirmation of the presence of Crab-eating Raccoon Procyon cancrivorus (Procyonidae) in the Colombian Amazon, hypothesis of distributional area, and comments on juvenile specimens. Small Carnivore Conservation. 52 \& 53: 84-92.

Oliveira, T. G. (2006). Research in terrestrial Carnivora from Brazil: Current knowledge and priorities for the new millennium. En Morato, R. G, Rodrigues, F.H.G., Eizirik, E., Mangini, P.R., Azevedo, F.C.C. \& MarinhoFilho, J. (editores). Manejo e Conservação de Mamíferos neotropicais. p. 39-45. São Paulo: Ibama

Orjuela, C., O.J. \& Jiménez, G. (2004). Estudio de la abundancia relativa para mamíferos en diferentes tipos de coberturas y carretera, finca Hacienda Cristales, área Cerritos, La Virginia, municipio de Pereira, departamento de Risaralda, Colombia. Universitas Scientiarum. 9: 87-96.

Overstreet, R.M. (1970). Baylisascaris procyonis (Stefansky and Zarnowiski, 1951) from the kinkajou, Potos flavus in Colombia. Proceedings of the Helminthological Society of Washington. 37: 192-195.

Páez, A., Saad, C., Núñez, C., Boshell, J. (2005). Molecular epidemiology of rabies in northern Colombia 1994-2003. Evidence for human and fox rabies associated with dogs. Epidemiology and Infection. 133: 529-536.

Pardo, L.E., \& Payán, E. (2015). Mamíferos de un agropaisaje de palma de aceite en las sabanas inundables de Orocué, Casanare, Colombia. Biota Colombiana. 16: 54-66.

Payán, E. (2009). Hunting sustainability, species richness and carnivore conservation in Colombian Amazonia. PhD thesis. University College London, London.

Payán, E. \& Escudero, S. (2016) Densidad de jaguares (Panthera onca) y abundancia de grandes mamíferos terrestres en un área no protegida del Amazonas colombiano. En E. Payán, C. A. Lasso \& C. Castaño-Uribe (editores). Conservación de grandes vertebrados en áreas no protegidas de Colombia, Venezuela y Brasil. p. 225-242. Bogotá: Instituto Alexander von Humboldt.

Ramírez-Cháves, H.E. \& Noguera-Urbano, E.A. (2011). Lista preliminar de los mamíferos (Mammalia: Theria) del departamento de Nariño, Colombia. Biota Colombiana. 11: 117-140.

Ramírez-Cháves, H. E. \& Pérez, W. A. (2015). New record of crab-eating fox Cerdocyon thous in Southwestern Colombia with comments on its distribution in Colombia and Ecuador. Canid Biology \& Conservation. 18: 6-9.

Ramírez-Cháves, H. E., Pérez, W., Ramírez-Mosquera, J. (2008). Mamíferos presentes en el municipio de Popayán, Cauca-Colombia. Boletín Científico Museo de Historia Natural Universidad de Caldas. 12: 65-89.

Ramírez-Cháves H.E., Cháves-Salazar, J.M., Mendoza-Escobar, R.E. (2013). Nuevo registro del lobo de páramo Lycalopex culpaeus (Mammalia: Canidae) en el suroccidente de Colombia con notas sobre su distribución en el país. Acta Zoológica Mexicana. 29 (2): 412-422.

Rangel-Ch., J.O. \& Aguilar-P, M. (1995). Una aproximación sobre la diversidad climática en las regiones naturales de Colombia. En: J.O. Rangel-Ch. (editor). Colombia Diversidad Biótica I. Instituto de Ciencias Naturales p. 2577. Universidad Nacional de Colombia.

Regan, T.J., Burgman, M.A., McCarthy, M.A., Master, L.L., Keith, D.A., Mace, G.M., Andelman, S.J. (2005). The consistency of extinction risk classification protocols. Conservation Biology. 19: 1969-1977.

Reid, F. \& Helgen, K. (2008). Nasuella olivacea. The IUCN Red List of Threatened Species. Version 2014.2. Disponible en: www.iucnredlist.org. Fecha de consulta: 7 de octubre, 2014.

Rodríguez, A. (1995). Rango de acción y hábitat alimenticio del coatí de montaña (Nasuella olivacea) en la Reserva Biológica Carpanta (Cundinamarca). (Tesis de pregrado). Universidad Distrital Francisco José de Caldas. Bogotá D. C.

Rodríguez-Rojas, C. (2005). Abundancia relativa de mamíferos en dos tipos de cobertura vegetal en la margen nororiental del santuario de flora y fauna Otún, Quimbaya, Risaralda. (Tesis de pregrado). Pontificia Universidad Javeriana. Bogotá D.C.

Rodríguez-Bolaños A., Sánchez, P., Cadena, A. (2003). Patterns of activity and home range of Mountain Coati Nasuella olivacea. Small Carnivore Conservation. 29: 16-19.

Rodríguez-Bolaños, A., Cadena, A., Sánchez, P. (2000). Trophic characteristics in social groups of the Mountain Coati, Nasuella olivacea (Carnivora: Procyonidae). Small Carnivore Conservation. 23: 1-5.

Rodríguez-Mahecha, J.V., Alberico, M., Trujillo, F., Jorgenson, J. (2006). Libro Rojo de los Mamíferos de Colombia. Serie Libros Rojos de Especies Amenazadas de Colombia. Bogotá D.C., Colombia. Conservación Internacional Colombia, Ministerio de Ambiente, Vivienda y Desarrollo Territorial.

Rojas-Arbeláez, L.V., Rodríguez-Ruiz, V., Árias-Bernal, L., Rodríguez-Álvarez, C., Pereira-Bengoa, V.E. (2008). Determinación de anticuerpos de leptospira [sic.] spp. en pequeños mamíferos no voladores en un fragmento de bosque andino en la montaña del Zoológico Jaime Duque. Revista de Medicina Veterinaria. 16: 27-42.

Ruiz-García, M., Lichilín-Ortiz, N., Jaramillo, M.F. (2013). Molecular phylogenetics of two Neotropical carnivores, Potos flavus (Procyonidae) and Eira barbara (Mustelidae): No clear existence of putative morphological subspecies. En: M. Ruiz-García \& Shostell, J.M. (editores). Molecular population genetics, evolutionary biology, and biological conservation of Neotropical carnivores. p. 37-84.New York, Estados Unidos: Nova Publishers.

Saavedra-Rodríguez, C.A. \& Velandia-Perilla, J.H. (2011). Bassaricyon gabbii Allen, 1876 (Carnivora: Procyonidae): New distribution point on western range of Colombian Andes. Check List. 7: 505-507.

Sánchez, F., Sánchez-Palomino, P., Cadena, A. (2008). Species richness and indices of abundance of medium-sized mammals in Andean forest and reforestations with Andean alder: A preliminary analysis. Caldasia. 30: 197-208.

Sánchez-Lalinde, C. \& J. Pérez-Torres. (2008). Uso de hábitat de carnívoros simpátricos en una zona de bosque seco tropical de Colombia. Mastozoología Neotropical. 15: 67-74. 
Sánchez-Londoño, J.C. (2014) Uso compartido de una letrina por el zorro Cerdocyon thous y perros domésticos Canis familiaris (Carnivora: Canidae) en una zona periurbana del Valle de Aburrá (Antioquia, Colombia). Mammalogy Notes. 1: 12-14.

Solari, S., Muñoz-Saba, Y., Rodríguez-Mahecha, J.V., Defler, T.R, Ramírez-Cháves, H.E., Trujillo, F. (2013). Riqueza, endemismo y conservación de los mamíferos de Colombia. Mastozoología Neotropical. 20: 301-365.

Stevenson, P.R., Pérez-Torres, J., Muñoz-Saba, Y. (2006). Estado de conocimiento sobre los mamíferos terrestres y voladores en Colombia. Tomo II. En: Cháves, M.E. y Santamaría, M. (editores). 2006. Informe sobre el avance en el conocimiento y la información de la biodiversidad 1998 -2004. p. 151-169. Instituto de Investigación de Recursos Biológicos Alexander von Humboldt. Bogotá D.C., Colombia. 2 Tomos.

Suárez-Castro, A.F., Pinto-Sarmiento, I.D., García-Villalba, J.E. (2015).Familia Canidae. En: Suárez-Castro, A.F. \& Ramírez-Cháves (editores). Los carnívoros terrestres y semiacuáticos continentales de Colombia. Guía de campo. p. 56-77. Bogotá: Editorial Universidad Nacional de Colombia.

Suárez-Castro, A.F. \& Ramírez-Cháves, H.E. (editores) (2015). Los carnívoros terrestres y semiacuáticos continentales de Colombia. Guía de campo. Bogotá: Editorial Universidad Nacional de Colombia. 220 p.

Thatcher, V. (1971). Some Hookworms of the genus Ancylostoma from Colombia and Panama. The Helminthological Society of Washington. 36: 79-116.
Thomas O. (1909). Notes on some South American mammals, with descriptions of new species. Annals and Magazine of Natural History. 4 (8): 230-242.

Thomas O. (1912). Two new species of Nasua. Annals and Magazine of Natural History. 8 (10): 228-230.

Thomas O. (1914). New Nasua, Lutra, and Proechimys from South America. Annals and Magazine of Natural History. 8 (14): 57-61.

Thomas, O. (1918). A new wild dog from the Bogota cordillera. Annals and Magazine of Natural History. 13: 345-363.

Thomas, O. (1927). A new subspecies of Bassaricyon from Colombia. Annals and Magazine of Natural History. 20 (9): 80.

Torres-Mejía, A. \& De La Fuente, J. (2006). Risks associated with ectoparasites of wild mammals in the Department of Quindío, Colombia. The International Journal of Applied Research in Veterinary Medicine. 4: 187- 192.

Trapido, H. \& Sanmartín, C. (1971). Pichindé virus a new virus of the Tacaribe group from Colombia. American Journal of Tropical Medicine and Hygiene. 20: 631-641.

Walker, R. S., Novaro, A. J., Nichols, J. D. (2000). Consideraciones para la estimación de abundancia de poblaciones de mamíferos. Mastozoología Neotropical. 7 (2): 73-80.

Wells, E.A., D’Alessandro, A., Morales, G.A., Angel, D. (1981). Mammalian wildlife diseases as hazards to man and livestock in an area of the Llanos Orientales of Colombia. Journal of Wildlife Diseases. 17: 153-162. 